\title{
Do Failure of Posterior Tibial Nerve Stimulation Precludes to Use Sacral Neuromodulation in Patient With Overactive Bladder?
}

\author{
Louis-Paul Berthelot ${ }^{1}$, Benoit Peyronnet ${ }^{2}$, Jean-Nicolas Cornu ${ }^{3}$, Annabelle Aublé ${ }^{3}$, Elena Brassart ${ }^{1}$ Pierre Bigot $^{1}$, Julie Carrouget ${ }^{1}$ \\ ${ }^{1}$ Department of Urology, Angers University Hospital, Angers, France \\ ${ }^{2}$ Department of Urology, Rennes University Hospital, Rennes, France \\ ${ }^{3}$ Department of Urology, Rouen University Hospital, Rouen, France
}

Purpose: To evaluate the outcomes of sacral neuromodulation (SNM) after failure of transcutaneous posterior tibial nerve stimulation (TPTNS) in patients with overactive bladder (OAB).

Methods: A retrospective study was conducted in 3 university hospitals and included all patients with $\mathrm{OAB}$ and treated with SNM after TPTNS had been tried between October 2008 and May 2018. The primary endpoint was the proportion of definitive SNM device implantation in patients with $50 \%$ objective and/or subjective improvement after a test period (stage 1 ). The secondary outcomes of interest were changes of the number of diurnal voids and nocturia episodes per 24 hours between the end of TPTNS and the end of stage 1.

Results: Overall, 28 of the 43 patients included achieved at least $50 \%$ objective and/or subjective improvement during stage 1 and underwent an Interstim II implantation (65.1\%). The mean daytime frequency decreased significantly from 10.3/day at the end of TPTNS to 7.8 diurnal voids/day at the end of SNM stage $1(\mathrm{P}=0.01)$. The mean number of nocturia episodes decreased from 2.5/night at the end of TPTNS to 2.1/night at the end of stage 1, but this did not reach statistical significance $(\mathrm{P}=0.18)$. There was no other parameter significantly associated with response to $\mathrm{SNM}$

Conclusions: SNM might improve OAB symptoms in most patients who experienced no or poor efficacy with TPTNS. History of failed TPTNS should not preclude the use of SNM in OAB patients.

Keywords: Overactive bladder; Failure; Transcutaneous tibial nerve stimulation; Sacral neuromodulation; Outcome

- Research Ethics: This study was approved by the Institutional Review Board of the Commission Nationale de l'Informatique et des Libertés (No. 2216079)

- Conflict of Interest: No potential conflict of interest relevant to this article was reported.

\section{INTRODUCTION}

The global prevalence of overactive bladder $(\mathrm{OAB})$ in France has been estimated to be $14.4 \%$ in 2016 [1] and some predicted it could reach $20 \%$ in the worldwide population in 2018 [2]. This is a public health concern because it affects patients' quality of life and is a significant economic burden [3].
Various algorithms of management have been proposed in the past years; current French and international guidelines recommend: behavioral therapy and lifestyle changes as a first-line treatment, then pharmacological treatments (i.e., anticholinergics or beta-3 adrenergic receptor agonists) as second lines therapeutic options. Botulinum toxin A intradetrusor injections (BoNT), sacral neuromodulation (SNM), or posterior tibial

Corresponding author: Louis-Paul Berthelot (iD https://orcid.org/0000-0002-8927-3202 Department of Urology, Angers University Hospital, 4 rue Larrey, 49100 Angers, France

E-mail: dr.berthelot.louispaul@gmail.com / Tel: +33681512519 / Fax: +33241353637

Submitted: June 4, 2019 / Accepted after revision: September 29, 2019 
nerve stimulation (PTNS) is the third-line therapeutic options before invasive surgery [4-6].

Percutaneous PTNS (PPTNS) has been proven effective in patients with $\mathrm{OAB}$ in several randomized controlled trials versus sham, anticholinergics, and lifestyle modification [7]. A percutaneous needle is used by the care provider to stimulate the tibial nerve once weekly. Because of its relative invasiveness, it has been considered as a third-line therapy. Fifteen years ago, a less invasive and cheaper method was proposed to stimulate the posterior tibial nerve using 2 surface electrodes.

Transcutaneous PTNS (TPTNS) has recently been shown to be noninferior compared to PPTNS [8]. Many prospective studies have demonstrated TPTNS efficacy and excellent safety profile [9-11]. In many Europeans centers TPTNS is offered earlier than PPTNS in the OAB therapeutic algorithm.

When TPTNS fails, BoNT or SNM are considered as a thirdline therapy. Both are supported by high-level of evidence studies and the ROSETTA (Refractory Overactive Bladder: Sacral Neuromodulation vs Botulinum Toxin Assessment) trial has shown that they have similar efficacy at 2 years [12].

The mechanisms of action of PTNS and SNM are not fully elucidated but both are assumed to modulate bladder afferent signaling and central neural control of the micturition reflex $[13,14]$. In daily practice, some urogynaecologists are reluctant to offer SNM when PTNS has failed, assuming that it could have low chance of success by relying on similar mechanisms of action. However, no study has yet aimed to assess the outcomes of SNM in patients who did not respond to PTNS [15]. The objective of this study was to evaluate the outcomes of SNM in patients previously treated with TPTNS.

\section{MATERIALS AND METHODS}

\section{Study Design}

After Institutional Review Board approval (Commission Nationale de l'Informatique et des Libertés, No. 2216079), we conducted a retrospective study in 3 French University Hospitals. The patients included were above 18 years of age, and had $\mathrm{OAB}$ symptoms treated between October 2008 and May 2018 initially with TPTNS and subsequently with SNM. We excluded patients with previous surgery for $\mathrm{OAB}$ (i.e., SNM or BoNT) and other indications of SNM (bladder pain syndrome, nonobstructive urinary retention, and fecal incontinence). TPTNS was used as first-line therapy or second-line therapy after failure of pharmacological treatments. SNM was offered after
TPTNS at the physicians' discretion because of either lack of TPTNS efficacy, difficulty of TPTNS utilization or patients' preference.

\section{Pretreatment Evaluation}

All patients had a complete urological investigation before treatment with TPTNS. The following data were recorded: past medical history and physical examination, number of pads, bladder diary data. The severity of symptoms was evaluated with the Urinary Symptom Profile (USP) score and the USP$\mathrm{OAB}$ subscore [1]. All patients underwent urodynamics before TPTNS initiation.

\section{Transcutaneous Posterior Tibial Nerve Stimulation Technique}

TPTNS was performed with a UROstim Schwa medico 2 (Schwa-medico France, Rouffach, France) in all departments. The patients were taught how to use the device by a urology nurse specialist in a 1-hour session. After this session, they carried out TPTNS at home by themselves.

The device is composed of a stimulator connected to 2 surface electrodes (diameter, $50 \mathrm{~mm}$ ) positioned $5 \mathrm{~cm}$ above the medial malleolus and $2 \mathrm{~cm}$ over the path of the tibial nerve. An electric bipolar stimulation was set to a frequency of $10 \mathrm{~Hz}$ and an impulsion of 150 micros for 20 minutes once or twice daily.

\section{Follow-up}

Patients were seen at 3 and 6 months to assess treatment efficacy based on bladder diary, physical examination and USP score. There was no repeat urodynamic investigation. Depending upon treatment's efficacy and patient's compliance, TPTNS was either stopped or continued after these 2 visits.

\section{Sacral Neuromodulation Technique}

Staged implant was used for all SNM. During stage 1, under general anesthesia, the S3 nerve root was stimulated with a tinned lead placed under fluoroscopic guidance. When a satisfactory motor response was obtained, the tinned lead was connected with a temporary external stimulator device for a test period of seven to 14 days.

Patients who achieved at least 50\% objective (USP score, bladder diary) and/or subjective improvement were implanted with the SNM definitive device (InterStim II, Medtronic, Minneapolis, MN, USA). 


\section{Follow-up}

Postoperative complications after stages 1 and 2 were recorded. Patients were evaluated clinically on a regular basis with no standardized follow-up protocol.

\section{Outcomes}

The following patients' characteristics were collected: sex, age, bladder diary, and urodynamic parameters.

The primary endpoint was the proportion of patients who achieved at least $50 \%$ objective and/or subjective improvement during stage 1 and underwent Interstim II implantation. The secondary outcomes of interest were change of the number of diurnal voids and nocturia episodes per 24 hours between the end of TPTNS and stage 1.

\section{Statistical Analysis}

Means and standard deviations were reported for continuous variables, and proportions for nominal variables. Comparisons between groups were performed using the $\chi^{2}$ test or Fisher exact test for discrete variables, and Mann-Whitney test for continuous variables as appropriate. Change of continuous variables over time was assessed using the paired Student $\mathrm{t}$-test and change of categorical variables over time was assessed using the McNemar test. Statistical analyses were performed using JMP v.12.0 software (SAS Institute Inc., Cary, NC, USA). All tests were 2 -sided with a level of $\mathrm{P}<0.05$ considered statistically significant.

\section{RESULTS}

\section{Patient's Characteristics}

Over the study period, 50 patients were treated with TPTNS before SNM; 6 were excluded from the final analysis because they had a neurologic condition and 1 patient because the indication for TPTNS was not OAB but nonobstructive urinary retention. The patients' characteristics are summarized in Table 1. There were 35 women (81.4\%) and the mean age of $71.4 \pm 9.2$ years. Twenty-five patients had detrusor overactivity on pretherapeutic urodynamic testing $(58.1 \%)$ and the mean maximum cystometric capacity was $229.3 \pm 116.2 \mathrm{~mL}$. On baseline bladder diary, the mean numbers of diurnal voids $/ 24 \mathrm{hr}$, of urgency/24 hr and of nocturia episodes/24 hr were $10.8 \pm 4.3$, $3.8 \pm 3.1$, and $2.8 \pm 1.8$, respectively. The mean USP score (/39) and USP-OAB subscore (/21) at baseline were $18.5 \pm 6.3$ and $12.3 \pm 3.6$, respectively. TPTNS was used as the first-line $\mathrm{OAB}$
Table 1. Patients' characteristics $(n=43)$

\begin{tabular}{lc}
\hline Characteristic & Value \\
\hline Age $(\mathrm{yr})$ & $71.4(62.3-80.6)$ \\
Female sex & $35(81.4)$ \\
Daytime frequency & $10.8(8.6-13)$ \\
No. of nocturia episodes & $2.8(1.9-3.7)$ \\
Coexistent voiding symptoms & $4(9.3)$ \\
Maximum cystometric capacity $(\mathrm{mL})$ & $229.7(171.6-287.8)$ \\
Detrusor overactivity & $25(58.1)$ \\
Mean maximum flow rate $(\mathrm{mL} / \mathrm{sec})$ & $17.3(12.8-21.8)$ \\
Postvoid residual $(\mathrm{mL})$ & $21.6(2.8-40.5)$ \\
USP score $(/ 39)$ & $18.5(15.3-21.6)$ \\
USP-OAB subscore $(/ 21)$ & $12.3(10.5-14.1)$ \\
\hline
\end{tabular}

Values are presented as mean (range) or number (\%).

treatment in 4 patients (9.3\%). All other patients had failed at least one $\mathrm{OAB}$ medication (antimuscarinic and/or beta3-agonist) and/or behavioral therapy.

\section{TPTNS Outcomes}

Overall, TPTNS was poorly effective. After a median 169.5 days of TPTNS daily use, none of the patients had achieved a 50\% improvement of OAB symptoms according to the USP-OAB subscore. The USP-OAB subscore decreased by $2.9 \%$ on average between the pretreatment visit and the last TPTNS visit, from 12.4 to 11.8 which was not statistically significant $(\mathrm{P}=$ 0.27). The USP score decreased from 18.1 at baseline to 17.3 at the last TPTNS visit which was not statistically significant $(\mathrm{P}=0.37)$. The mean daytime frequency did not decrease significantly, being 11.1/day at baseline and 10.3/day at the last TPTNS visit $(\mathrm{P}=0.12)$. Similarly, the mean number of nocturia episodes remained relatively stable, being $2.8 /$ night at baseline and 2.5/night at the last TPTNS visit $(\mathrm{P}=0.61)$. The mean number of urgency/24 hr did not change significantly being 3.8/day at baseline and 5/day at the last TPTNS visit $(\mathrm{P}=0.53)$.

\section{SNM Outcomes}

Overall, 28 of the 43 patients included achieved at least $50 \%$ objective and/or subjective improvement during stage 1 and underwent an Interstim II implantation (65.1\%). The mean daytime frequency decreased significantly from 10.3/day at the end of TPTNS to 7.8 diurnal voids/day at the end of SNM stage 1 $(\mathrm{P}=0.01)$ (Fig. 1). The mean number of nocturia episodes decreased from 2.5/night at the end of TPTNS to 2.1/night at the 


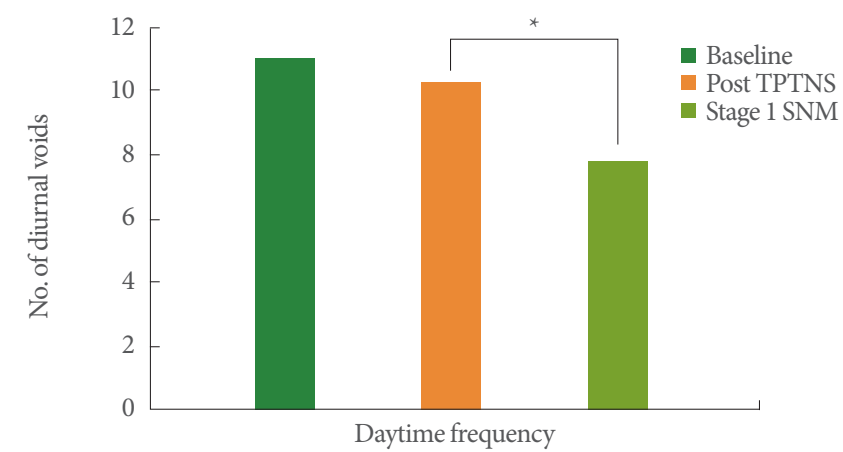

Fig. 1. Change of daytime frequency over consecutive stimulations. TPTNS, transcutaneous posterior tibial nerve stimulation; SNM, sacral neuromodulation. ${ }^{\star} \mathrm{P}<0.05$.

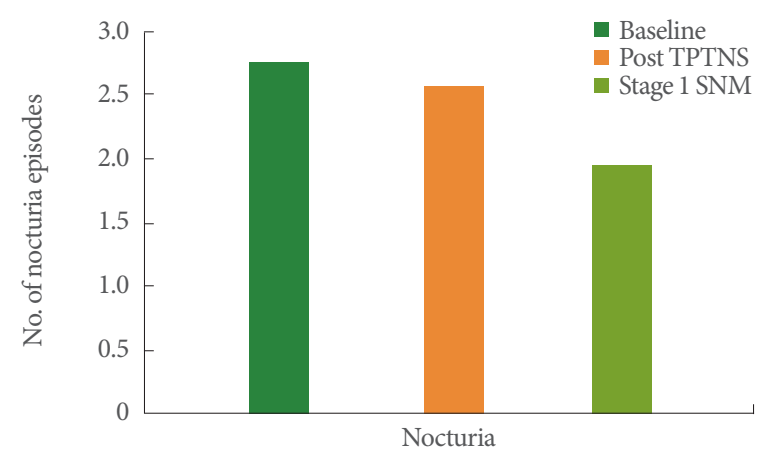

Fig. 2. Change of nocturia's episodes over consecutive stimulations. TPTNS, transcutaneous posterior tibial nerve stimulation; SNM, sacral neuromodulation.

Table 2. Univariate comparison of patients with sacral neuromodulator implanted vs. not

\begin{tabular}{lccc}
\hline Variable & $\begin{array}{c}\text { Patients with no Interstim II } \\
\text { implanted (n=15) }\end{array}$ & $\begin{array}{c}\text { Patients with Interstim II } \\
\text { implanted (n=28) }\end{array}$ & P-value \\
\hline Age (yr) & $72.2(65.3-79.1)$ & $72.5(62.9-82.2)$ & 0.84 \\
Female sex & $12(80)$ & $23(82.1)$ & 0.99 \\
Daytime frequency & $9.5(7.7-11.2)$ & $11.6(9.2-13.9)$ & 0.06 \\
No. of nocturia episodes & $2.4(1.6-3.1)$ & $3(2.1-4)$ & 0.28 \\
Cystometric capacity (mL) & $267.33(200.2-334.4)$ & $211(160.3-262)$ & 0.22 \\
Detrusor overactivity & $7(46.7)$ & $18(64.3)$ & 0.33 \\
Baseline postvoid residual (mL) & $18(2.3-33.7)$ & $19.4(16.2-22.6)$ & 0.86 \\
Baseline USP score & $17.3(14.1-20.4)$ & $13(11.2-14.7)$ & 0.47 \\
Mean baseline USP-OAB subscore & $11.4(9.5-13.3)$ & $142.4 \pm 68.5$ & 0.23 \\
Duration of TPTNS utilization (day) & $207.4 \pm 142.9$ & $2.5 \pm 0.3$ & 0.11 \\
Change of USP-OAB subscore at last TPTNS visit (\%) & $-15.1 \pm 0.2$ & 0.13 \\
\hline
\end{tabular}

USP, Urinary Symptom Profile questionnaire; OAB, overactive bladder; TPTNS, transcutaneous posterior tibial nerve stimulation.

end of stage 1, but this did not reach statistical significance $(\mathrm{P}=0.18)$ (Fig. 2). The mean number of urgency dwindled from 5/day at the end of the TPTNS courses to 2.4/day at the end of stage $1(\mathrm{P}=0.06)$. Two patients had their neuromodulator explanted due to loss of efficacy after 24 and 40 months respectively. After a median follow-up period of 22.5 months, 12 of the 28 implanted patients (42.9\%) had recurrence of their $\mathrm{OAB}$ symptoms and 7 elected to undergo a subsequent $\mathrm{OAB}$ treatment: 4 received botulinum toxin $\mathrm{A}$ intradetrusor injections, 1 switched back to antimuscarinics, 1 to beta-3 agonists, and 1 to TPTNS.

\section{Adverse Events}

No patients stopped TPTNS treatment because of side effects and only 2 because of difficulty in handling the device. The sacral neuromodulator was explanted in 2 patients because of infection.

\section{Predictive Factors of SNM Efficacy}

Patients who achieved at least $50 \%$ objective and/or subjective improvement during stage 1 and underwent an Interstim II implantation tended to have smaller improvement with TPTNS as assessed by the USP-OAB subscore ( $2.5 \%$ vs. $-15.1 \%, \mathrm{P}=0.13$ ) (Table 2) and a shorter duration of TPTNS utilization (142.4 days vs. 207.4 days, $\mathrm{P}=0.11$ ). Patients who underwent Interstim II implantation tended to have more severe daytime frequency at baseline (11.6 vs. 9.5, $\mathrm{P}=0.06$ ). There was no other parameter significantly associated with response to SNM (Table 2). 


\section{DISCUSSION}

Our study is the first to assess the outcomes of SNM in OAB patients previously treated with PTNS. We found that SNM can provide significant improvement in patients who failed TPTNS and did not evidence statistically significant association between the response to TPTNS and the efficacy of SNM.

PTNS has become over the past decade a well-accepted third-line therapeutic option in patients with $\mathrm{OAB}[4,6,16]$. The stimulation could be delivered to the posterior tibial nerve through 2 distinct routes: using a fine needle (PPTNS) or using a surface electrode (TPTNS). While sham-controlled randomized trial supporting the use of each of these 2 approaches for $\mathrm{OAB}$ do exist, the worldwide spread of transcutaneous-PTNS remains more limited despite significant possible advantages such as lower invasiveness and costs. Our cohort confirmed the excellent safety profile of TPTNS with no adverse events noted, no withdrawal for tolerance issues and only 2 discontinuations because of difficulty in handling the TPTNS device. Over the past decade, a growing number of randomized controlled trials have demonstrated the efficacy of TPTNS in patients with OAB [7]. Recently Ramírez-García et al. [8] showed that TTNS is not inferior than PTNS. As a result of these compelling evidence and of its noninvasiveness TPTNS is likely to move earlier in the $\mathrm{OAB}$ therapeutic algorithm as other studies already suggest $[7,8,17]$. Hence it is possible that clinicians face an increasing number of OAB patients who failed TPTNS and that the clinical issue addressed here, i.e., is SNM a relevant third-line therapy in this patients' population, becomes relatively common in daily practice.

In our study, 28 patients (65.1\%) had a definitive implantation of SNM after stage 1. While this implantation rate is slightly lower than the one from the Insite trial [18], similar rates of implantation have been found in other "real life" series [14]. The other outcomes we observed with significant decrease in daytime frequency and nocturia and a maintained improvement in about $60 \%$ of patients after a median follow-up period of 22.5 months is in line with other SNM series and suggest that SNM might be as effective in nonresponders to TPTNS as in other patients' populations.

Currently, the mechanisms of action of TTNS and SNM are not well known [19]. Both treatments act on afferent pathways from the bladder [20] and might act through a similar mechanism of neuronal modulation, however the absence of a correlation between TTNS and SNM efficacy in our series seems to contradict this theory. Weissbart et al. [21] recently demonstrated a change of brain activity after SNM and 10 years ago, Finazzi-Agrò et al. [22] demonstrated a modification of cerebral plasticity after tibial nerve stimulation. Both had an impact on the central nervous system. One could hypothesize that SNM effect could add up to PTNS, further modulating central nervous system plasticity. In such a theory, TPTNS could be regarded as a "neoadjuvant" noninvasive therapy maximizing the chance of success of SNM. However, our findings would suggest the opposite. The tendency towards lower response to TPTNS of patients who were later implanted with Interstim II would indicate that TPTNS and SNM might rely on similar mechanisms of action and that effective TPTNS may diminish the amplitude of effect of SNM. Hence, hypothetically, some patients might not reach the 50\% improvement threshold and be denied SNM implantation while they could have experienced greater improvement during stage 1 if naive of any nerve stimulation. Further data are needed to elucidate the possible overlap in SNM and TPTNS effect and mechanisms of action.

Our study did not include a proper control group treated with intradetrusor injections of botulinum toxin. This was mostly due to the relatively recent approval of intradetrusor botulinum toxin injections for OAB in France (June 2014) and inherent limited number of patients treated with botulinum toxin after TPTNS in the 3 participating institutions. Intradetrusor botulinum toxin injections having become the direct contender of SNM as first-line therapies for OAB, data on its efficacy would be of interest even though the very different mode of action of TPTNS and intradetrusor botulinum toxin would make overlapping lack of efficacy very unlikely.

Our study has several limitations that should be acknowledged. Current guidelines recommend that Interstim II should be implanted in patients with a $50 \%$ objective and/or subjective improvement after stage 1 [14]. We used this outcome as a surrogate of SNM efficacy which might be regarded as a drawback. Unfortunately, as in most retrospective series, some data were missing which prevents to assess the impact of SNM using validated questionnaires. The lack of other parameters assessing objective and subjective improvement or quality of life scores over post SNM implantation follow-up was a limitation of the present study. Our study did not include a proper control group (e.g., treated with intradetrusor injections of botulinum toxin) which might be regarded as a limitation. This was mostly due to the relatively recent approval of intradetrusor botulinum toxin injections for OAB in France (June 2014) and inherent 
limited number of patients treated with botulinum toxin after TPTNS in the 3 participating institutions. To determine the efficacy of TPTNS, we used the USP score. This score was developed in France [23] and was later validated in English but has not been widely adopted in the literature and this might be seen as a limitation of our study. Another shortcoming of our series was the limited sample size and inherent lack of statistical power which limits the conclusions that could be drawn from the present study. We did not perform an a priori sample size calculation because of the retrospective study design. Finally, one of the possible issues with TPTNS, which is done by the patient himself at home is lack of patients' compliance. Data on patients' compliance with TPTNS can be extracted from the UROstim 2 device but were not available in the present cohort which could be seen as a study's limitation as some of nonresponders to TPTNS may well have been poorly compliant patients.

\section{AUTHOR CONTRIBUTION STATEMENT}

- Full access to all the data in the study and takes responsibility for the integrity of the data and the accuracy of the data analysis: $L P B$

- Study concept and design: $L P B, B P$

- Acquisition of data: $L P B, A A$, JC

- Analysis and interpretation of data: $L P B, A A, J N C, B P$

- Drafting of the manuscript: $L P B, J C, P B, B P$

- Critical revision of the manuscript for important intellectual content: $L P B$

- Statistical analysis: $L P B$

- Obtained funding: $L P B, E B$

- Administrative, technical, or material support: $L P B$

- Study supervision: $L P B$

\section{REFERENCES}

1. Cornu JN, Amarenco G, Bruyere F, Chartier-Kastler E, Fatton B, Grise P, et al. Prevalence and initial management of overactive bladder in France: a cross-sectional study. Prog Urol 2016;26:41524.

2. Irwin DE, Kopp ZS, Agatep B, Milsom I, Abrams P. Worldwide prevalence estimates of lower urinary tract symptoms, overactive bladder, urinary incontinence and bladder outlet obstruction. BJU Int 2011;108:1132-8.

3. Irwin DE, Mungapen L, Milsom I, Kopp Z, Reeves P, Kelleher C.
The economic impact of overactive bladder syndrome in six Western countries. BJU Int 2009;103:202-9.

4. Gormley EA, Lightner DJ, Faraday M, Vasavada SP; American Urological Association; Society of Urodynamics, Female Pelvic Medicine. Diagnosis and treatment of overactive bladder (nonneurogenic) in adults: AUA/SUFU guideline amendment. J Urol 2015;193:1572-80.

5. Corcos J, Przydacz M, Campeau L, Gray G, Hickling D, Honeine C, et al. CUA guideline on adult overactive bladder. Can Urol Assoc J 2017;11:E142-73.

6. Nambiar AK, Bosch R, Cruz F, Lemack GE, Thiruchelvam N, Tubaro A, et al. EAU guidelines on assessment and nonsurgical management of urinary incontinence. Eur Urol 2018;73:596-609.

7. Booth J, Connelly L, Dickson S, Duncan F, Lawrence M. The effectiveness of transcutaneous tibial nerve stimulation (TTNS) for adults with overactive bladder syndrome: a systematic review. Neurourol Urodyn 2018;37:528-41.

8. Ramírez-García I, Blanco-Ratto L, Kauffmann S, Carralero-Martínez A, Sánchez E. Efficacy of transcutaneous stimulation of the posterior tibial nerve compared to percutaneous stimulation in idiopathic overactive bladder syndrome: randomized control trial. Neurourol Urodyn 2019;38:261-8.

9. Ammi M, Chautard D, Brassart E, Culty T, Azzouzi AR, Bigot P. Transcutaneous posterior tibial nerve stimulation: evaluation of a therapeutic option in the management of anticholinergic refractory overactive bladder. Int Urogynecol J 2014;25:1065-9.

10. de Sèze M, Raibaut P, Gallien P, Even-Schneider A, Denys P, Bonniaud $V$, et al. Transcutaneous posterior tibial nerve stimulation for treatment of the overactive bladder syndrome in multiple sclerosis: results of a multicenter prospective study. Neurourol Urodyn 2011; 30:306-11.

11. Leroux PA, Brassart E, Lebdai S, Azzouzi AR, Bigot P, Carrouget J. Transcutaneous tibial nerve stimulation: 2 years follow-up outcomes in the management of anticholinergic refractory overactive bladder. World J Urol 2018;36:1455-60.

12. Amundsen CL, Komesu YM, Chermansky C, Gregory WT, Myers DL, Honeycutt EF, et al. Two-year outcomes of sacral neuromodulation versus onabotulinumtoxinA for refractory urgency urinary incontinence: a randomized trial. Eur Urol 2018;74:66-73.

13. Choudhary M, van Mastrigt R, van Asselt E. Effect of tibial nerve stimulation on bladder afferent nerve activity in a rat detrusor overactivity model. Int J Urol 2016;23:253-8.

14. Goldman HB, Lloyd JC, Noblett KL, Carey MP, Castaño Botero JC, Gajewski JB, et al. International Continence Society best practice statement for use of sacral neuromodulation. Neurourol Urodyn 
2018;37:1823-48.

15. Liberman D, Ehlert MJ, Siegel SW. Sacral neuromodulation in urological practice. Urology 2017;99:14-22.

16. Apostolidis A, Averbeck MA, Sahai A, Rahnama’i MS, Anding R, Robinson D, et al. Can we create a valid treatment algorithm for patients with drug resistant overactive bladder $(\mathrm{OAB})$ syndrome or detrusor overactivity (DO)? Results from a think tank (ICI-RS 2015). Neurourol Urodyn 2017;36:882-93.

17. Schreiner L, dos Santos TG, Knorst MR, da Silva Filho IG. Randomized trial of transcutaneous tibial nerve stimulation to treat urge urinary incontinence in older women. Int Urogynecol J 2010; 21:1065-70.

18. Siegel S, Noblett K, Mangel J, Griebling TL, Sutherland SE, Bird ET, et al. Results of a prospective, randomized, multicenter study evaluating sacral neuromodulation with InterStim therapy compared to standard medical therapy at 6-months in subjects with mild symptoms of overactive bladder. Neurourol Urodyn 2015;34:224-30.

19. Gajewski JB, Kanai AJ, Cardozo L, Ikeda Y, Zabbarova IV. Does our limited knowledge of the mechanisms of neural stimulation limit its benefits for patients with overactive bladder? ICI-RS 2013. Neurourol Urodyn 2014;33:618-21.

20. Leng WW, Chancellor MB. How sacral nerve stimulation neuromodulation works. Urol Clin North Am 2005;32:11-8.

21. Weissbart SJ, Bhavsar R, Rao H, Wein AJ, Detre JA, Arya LA, et al. Specific changes in brain activity during urgency in women with overactive bladder after successful sacral neuromodulation: a functional magnetic resonance imaging study. J Urol 2018;200:382-8.

22. Finazzi-Agrò E, Rocchi C, Pachatz C, Petta F, Spera E, Mori F, et al. Percutaneous tibial nerve stimulation produces effects on brain activity: study on the modifications of the long latency somatosensory evoked potentials. Neurourol Urodyn 2009;28:320-4.

23. Haab F, Richard F, Amarenco G, Coloby P, Arnould B, Benmedjahed $\mathrm{K}$, et al. Comprehensive evaluation of bladder and urethral dysfunction symptoms: development and psychometric validation of the Urinary Symptom Profile (USP) questionnaire. Urology 2008;71:646-56. 